\title{
Reduction formulae for the Lauricella functions in several variables
}

Junesang Choi ${ }^{1 *}$ and Arjun K Rathie ${ }^{2}$

\section{"Correspondence:}

junesang@mail.dongguk.ac.kr

1 Department of Mathematics,

Dongguk University, Gyeongju,

Korea

Full list of author information is

available at the end of the article

\begin{abstract}
The main objective of this paper is to show how one can obtain several interesting reduction formulae for Lauricella functions from a multiple hypergeometric series identity established earlier by Jaimini et al. The results are derived with the help of generalized Kummer's second summation formulas obtained earlier by Lavoi et al. Some special cases of our main result are explicitly demonstrated.

MSC: Primary 33C70; 33C065; secondary 33C90; 33C05

Keywords: generalized hypergeometric function ${ }_{p} F_{q}$; gamma function; Pochhammer symbol; generalized Lauricella series; multiple series identities; Kummer's second summation theorem; generalized Kummer's second summation theorem
\end{abstract}

\section{Introduction and results required}

In the usual notation, let $\mathbb{C}$ denote the set of complex numbers. For

$$
\alpha_{j} \in \mathbb{C} \quad(j=1, \ldots, p) \quad \text { and } \quad \beta_{j} \in \mathbb{C} \backslash \mathbb{Z}_{0}^{-} \quad\left(\mathbb{Z}_{0}^{-}:=\mathbb{Z} \cup\{0\}=\{0,-1,-2, \ldots\}\right) \text {, }
$$

the generalized hypergeometric function ${ }_{p} F_{q}$ with $p$ numerator parameters $\alpha_{1}, \ldots, \alpha_{p}$ and $q$ denominator parameters $\beta_{1}, \ldots, \beta_{q}$ is defined by (see, for example, [1, Chapter 4$]$; see also [2, pp.71-72])

$$
\begin{aligned}
& { }_{p} F_{q}\left[\begin{array}{l}
\alpha_{1}, \ldots, \alpha_{p} ; \\
\beta_{1}, \ldots, \beta_{q} ;
\end{array}\right]=\sum_{n=0}^{\infty} \frac{\prod_{j=1}^{p}\left(\alpha_{j}\right)_{n}}{\prod_{j=1}^{q}\left(\beta_{j}\right)_{n}} \frac{z^{n}}{n !}={ }_{p} F_{q}\left(\alpha_{1}, \ldots, \alpha_{p} ; \quad \beta_{1}, \ldots, \beta_{q} ; \quad z\right) \\
& \left(p, q \in \mathbb{N}_{0}:=\mathbb{N} \cup\{0\}=\{0,1,2, \ldots\} ; p \leqq q+1 ; p \leqq q \text { and }|z|<\infty ;\right. \\
& p=q+1 \text { and }|z|<1 ; p=q+1,|z|=1 \text { and } \Re(\omega)>0),
\end{aligned}
$$

where

$$
\omega:=\sum_{j=1}^{q} \beta_{j}-\sum_{j=1}^{p} \alpha_{j} \quad\left(\alpha_{j} \in \mathbb{C}(j=1, \ldots, p) ; \beta_{j} \in \mathbb{C} \backslash \mathbb{Z}_{0}^{-}(j=1, \ldots, q)\right)
$$

\section{Springer}

(C) 2013 Choi and Rathie; licensee Springer. This is an Open Access article distributed under the terms of the Creative Commons Attribution License (http://creativecommons.org/licenses/by/2.0), which permits unrestricted use, distribution, and reproduction in any medium, provided the original work is properly cited. 
and $(\lambda)_{n}$ is the Pochhammer symbol defined (for $\lambda \in \mathbb{C}$ ), in terms of the familiar gamma function $\Gamma$, by

$$
(\lambda)_{n}:=\frac{\Gamma(\lambda+n)}{\Gamma(\lambda)}= \begin{cases}1 & (n=0), \\ \lambda(\lambda+1) \cdots(\lambda+n-1) & (n \in \mathbb{N})\end{cases}
$$

The generalized Lauricella series in several variables is defined and represented in the following manner (see, for example, [3, p.37]; see also [4]):

$$
\begin{aligned}
F_{C: D^{\prime} ; \ldots, D^{(n)}}^{A: B^{\prime}, \ldots ;(n)}\left(\begin{array}{c}
z_{1} \\
\vdots \\
z_{n}
\end{array}\right) \equiv & F_{C: D^{\prime}, \ldots ; D^{(n)}}^{A: B^{\prime}, \ldots, B^{(n)}}\left(\begin{array}{c}
{\left[(a): \theta^{\prime}, \ldots, \theta^{(n)}\right]:} \\
{\left[(c): \psi^{\prime}, \ldots, \psi^{(n)}\right]:}
\end{array}\right. \\
& {\left[\left(b^{\prime}: \phi^{\prime}\right)\right] ; \quad \ldots ; \quad\left[\left(b^{(n)}\right): \phi^{(n)}\right] ; } \\
& {\left[\left(d^{\prime}: \delta^{\prime}\right)\right] ; \quad \ldots ; \quad\left[\left(d^{(n)}\right): \delta^{(n)}\right] ; } \\
:= & \sum_{m_{1}, \ldots, m_{n}=0}^{\infty} \Lambda\left(m_{1}, \ldots, m_{n}\right) \frac{z_{1}^{m_{1}}}{m_{1} !} \ldots \frac{z_{n}^{m_{n}}}{m_{n} !},
\end{aligned}
$$

where, for convenience,

$$
\begin{aligned}
& \Lambda\left(m_{1}, \ldots, m_{n}\right) \\
& :=\frac{\prod_{j=1}^{A}\left(a_{j}\right)_{m_{1} \theta_{j}^{\prime}+\cdots+m_{n} \theta_{j}^{(n)}} \prod_{j=1}^{B^{\prime}}\left(b_{j}^{\prime}\right)_{m_{1} \phi_{j}^{\prime}} \cdots \prod_{j=1}^{B^{(n)}}\left(b_{j}^{(n)}\right)_{m_{n} \phi_{j}^{(n)}}}{\prod_{j=1}^{C}\left(c_{j}\right)_{m_{1} \psi_{j}^{\prime}+\cdots+m_{n} \psi_{j}^{(n)}} \prod_{j=1}^{D^{\prime}}\left(d_{j}^{\prime}\right)_{m_{1} \delta_{j}^{\prime}} \cdots \prod_{j=1}^{D^{(n)}\left(d_{j}^{(n)}\right)_{m_{n} \delta_{j}^{(n)}}^{(n)}}}
\end{aligned}
$$

the coefficients

$$
\begin{array}{ll}
\theta_{j}^{(k)} & (j=1, \ldots, A), \quad \phi_{j}^{(k)} \quad\left(j=1, \ldots, B^{(k)}\right), \quad \psi_{j}^{(k)} \quad(j=1, \ldots, C), \\
\delta_{j}^{(k)} & \left(j=1, \ldots, D^{(k)}\right) \quad(\forall k \in\{1, \ldots, n\}) ;
\end{array}
$$

are real and nonnegative, and (a) abbreviates the array of $A$ parameters $a_{1}, \ldots, a_{A} ;\left(b^{(k)}\right)$ abbreviates the array of $B^{(k)}$ parameters

$$
b_{j}^{(k)} \quad\left(j=1, \ldots, B^{(k)} ; \forall k \in\{1, \ldots, n\}\right),
$$

with similar interpretations for $\left(c^{(k)}\right)$, etc.

In the course of study of hypergeometric functions of two or more variables, Srivastava [5, 6], Buschman and Srivastava [7], Grosjean and Sharma [8] and Grosjean and Srivastava [9] established a large number of double and multiple series identities involving essentially arbitrary coefficients (see, for example, [10]). Later Jaimini et al. [11] presented three substantially more general multiple series identities involving similar coefficients, one of which is recalled here as in the following theorem (see [11, Theorem 3]). 
Theorem 1 Let $\Omega(m)$ represent a single-valued, bounded and real or complex function of the nonnegative integer-valued parameter $m$. Then we have

$$
\begin{aligned}
& \sum_{m_{1}, \ldots, m_{r}=0}^{\infty} \Omega\left(m_{1}+\cdots+m_{r}\right) \frac{(\alpha)_{m_{1}+m_{2}}}{(\alpha)_{m_{1}}(\alpha)_{m_{2}}} \prod_{j=1}^{r}\left\{\frac{\left(\mu_{j}\right)_{m_{j}}}{m_{j} !} x^{m_{j}}\right\} \\
& =\sum_{m, n=0}^{\infty} \Omega(m+2 n)\left(\mu_{1}+\cdots+\mu_{r}+2 n\right)_{m} \frac{\left(\mu_{1}\right)_{n}\left(\mu_{2}\right)_{n}}{(\alpha)_{n}} \frac{x^{m+2 n}}{m ! n !},
\end{aligned}
$$

provided that each of the series involved is absolutely convergent.

From Theorem 1, with

$$
\Omega(n)=\frac{\prod_{j=1}^{p}\left(a_{j}\right)_{n}}{\prod_{j=1}^{q}\left(b_{j}\right)_{n}} \quad\left(n \in \mathbb{N}_{0}\right)
$$

we arrive at the following multiple hypergeometric identity involving the generalized Lauricella function defined by (1.4) (see [11, Equation (3.1)]):

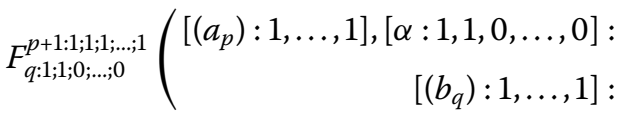

$$
\begin{aligned}
& \begin{array}{rrrr}
\left(\mu_{1}: 1\right) ; & \left(\mu_{2}: 1\right) ; \quad\left(\mu_{3}: 1\right) ; & \ldots ; & \left(\mu_{r}: 1\right) ; \\
(\alpha: 1) ; \quad(\alpha: 1) ; \quad \longrightarrow, x)
\end{array} \\
& =\sum_{n=0}^{\infty} \frac{\left(a_{1}\right)_{2 n} \cdots\left(a_{p}\right)_{2 n}}{\left(b_{1}\right)_{2 n} \cdots\left(b_{q}\right)_{2 n}} \frac{\left(\mu_{1}\right)_{n}\left(\mu_{2}\right)_{n}}{(\alpha)_{n}} \frac{x^{2 n}}{n !} \\
& { }_{p} F_{q}\left[\begin{array}{ll}
\mu_{1}+\cdots+\mu_{r}+2 n, & a_{1}+2 n, \ldots, a_{p}+2 n ; \\
& b_{1}+2 n, \ldots, b_{q}+2 n ;
\end{array} \quad x\right] .
\end{aligned}
$$

For $p=q=1,(1.7)$ reduces at once to (see [11, Equation (3.2)])

$$
\begin{aligned}
& F_{1: 1 ; i 1 ; 0 ; \ldots ; 0}^{2: 1 ; i 1, \ldots ; 1}\left(\begin{array}{r}
(a: 1, \ldots, 1),(\alpha: 1,1,0, \ldots, 0): \\
(b: 1, \ldots, 1):
\end{array}\right.
\end{aligned}
$$

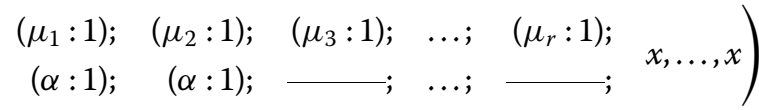

$$
\begin{aligned}
& =\sum_{n=0}^{\infty} \frac{(a)_{2 n}\left(\mu_{1}\right)_{n}\left(\mu_{2}\right)_{n}}{(b)_{2 n}(\alpha)_{n}} \frac{x^{2 n}}{n !}{ }_{2} F_{1}\left[\begin{array}{rr}
\mu_{1}+\cdots+\mu_{r}+2 n, & a+2 n ; \\
b+2 n ;
\end{array} \quad x\right] \text {. }
\end{aligned}
$$

Finally, if we use Kummer's second summation theorem (see, for example, [12, p.11, Equation 2.4(2)]; see also [13, Equation (1.4)])

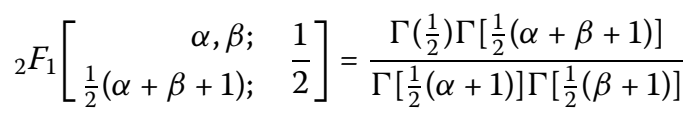


in (1.8) when

$$
x=\frac{1}{2} \quad \text { and } \quad b=\frac{1}{2}\left(a+\mu_{1}+\cdots+\mu_{r}+1\right)
$$

Jaimini et al. [11, Equation (3.6)] established the following interesting reduction formula for the generalized Lauricella function:

$$
\begin{aligned}
& F_{1: 1 ; 1 ; 0 ; \ldots ; 0 ; 0}^{2: 1 ; 1 ; \ldots ; 1}\left(\begin{array}{c}
(a: 1, \ldots, 1),(\alpha: 1,1,0, \ldots, 0): \\
\left(\frac{1}{2}\left(a+\mu_{1}+\cdots+\mu_{r}+1\right): 1, \ldots, 1\right):
\end{array}\right. \\
& \left.\left(\mu_{1}: 1\right) ; \quad\left(\mu_{2}: 1\right) ; \quad\left(\mu_{3}: 1\right) ; \ldots ; \quad\left(\mu_{r}: 1\right) ; \quad \frac{1}{2}, \ldots, \frac{1}{2}\right) \\
& (\alpha: 1) ; \quad(\alpha: 1) ; \quad \ldots ; \quad \longrightarrow \\
& =\frac{\Gamma\left(\frac{1}{2}\right) \Gamma\left[\frac{1}{2}\left(a+\mu_{1}+\cdots+\mu_{r}+1\right)\right]}{\Gamma\left[\frac{1}{2}\left(\mu_{1}+\cdots+\mu_{r}+1\right)\right] \Gamma\left[\frac{1}{2}(a+1)\right]} \\
& \left.\frac{a}{2}, \mu_{1}, \mu_{2} ; 1\right] .
\end{aligned}
$$

Here, in this paper, we aim mainly at showing how one can obtain several interesting reduction formulae for Lauricella functions from a multiple hypergeometric series identity (1.8). For this, we recall the following generalization of Kummer's second summation theorem (1.9) obtained earlier by Lavoie et al. [14]:

$$
\begin{aligned}
& { }_{2} F_{1}\left[\begin{array}{rr}
a, b ; & \frac{1}{2} \\
\frac{1}{2}(a+b+\ell+1) ; & 2
\end{array}\right] \\
& =\frac{\Gamma\left(\frac{1}{2}\right) \Gamma\left(\frac{a}{2}+\frac{b}{2}+\frac{\ell}{2}+\frac{1}{2}\right) \Gamma\left(\frac{a}{2}-\frac{b}{2}-\frac{\ell}{2}+\frac{1}{2}\right)}{\Gamma\left(\frac{a}{2}-\frac{b}{2}+\frac{|\ell|}{2}+\frac{1}{2}\right)} \\
& \cdot\left(\frac{A_{\ell}}{\Gamma\left(\frac{a}{2}+\frac{1}{2}\right) \Gamma\left(\frac{b}{2}+\frac{\ell}{2}+\frac{1}{2}-\left[\frac{\ell+1}{2}\right]\right)}+\frac{B_{\ell}}{\Gamma\left(\frac{a}{2}\right) \Gamma\left(\frac{b}{2}+\frac{\ell}{2}-\left[\frac{\ell}{2}\right]\right)}\right) \\
& (\ell=0, \pm 1, \pm 2, \pm 3, \pm 4, \pm 5) \text {, }
\end{aligned}
$$

where, and in what follows, $[x]$ denotes (as usual) the greatest integer less than or equal to $x$. The coefficients $A_{\ell}$ and $B_{\ell}$ are tabulated below.

It is remarked in passing that Equation (1.9) was incorrectly attributed to Gauss by Bailey [12, p.11, Equation 2.4(2)] (see, for details, [13, p.853]).

\section{Main reduction formulae}

The eleven reduction formulae in the form of a single result to be established are given in the following theorem.

Theorem 2 The following reduction formula holds true:

$$
\begin{aligned}
& F_{1: 1 ; 1 ; 0 ; \ldots ; 0}^{2: 1 ; 1 ; ; ; 1}\left[\begin{array}{r}
(a: 1, \ldots, 1),(\alpha: 1,1,0, \ldots, 0): \\
\left.\left(\frac{1}{2}\left(a+\mu_{1}+\cdots+\mu_{r}+\ell+1\right): 1, \ldots, 1\right)\right):
\end{array}\right. \\
& \left.\begin{array}{rrrrr}
\left(\mu_{1}: 1\right) ; & \left(\mu_{2}: 1\right) ; \quad\left(\mu_{3}: 1\right) ; & \ldots ; & \left(\mu_{r}: 1\right) ; & \frac{1}{2}, \ldots, \frac{1}{2}
\end{array}\right]
\end{aligned}
$$


Table 1 The coefficients $A_{\ell}$ and $B_{\ell}$

\begin{tabular}{lll}
\hline$\ell$ & $A_{\ell}$ & $B_{\ell}$ \\
\hline 5 & $-(b+a+6)^{2}+\frac{1}{4}(b-a+6)^{2}+\frac{1}{2}(b-a+6)(b+a+$ & $(b+a+6)^{2}-\frac{1}{4}(b-a+6)^{2}+\frac{1}{2}(b-a+6)(b+a+6)-$ \\
& $6)+11(b+a+6)-\frac{13}{2}(b-a+6)-20$ & $17(b+a+6)-\frac{1}{2}(b-a+6)+62$ \\
4 & $\frac{1}{2}(b+a+1)(b+a-3)-\frac{1}{4}(b-a+3)(b-a-3)$ & $-2(b+a-1)$ \\
3 & $-\frac{1}{2}(3 a+b-2)$ & $\frac{1}{2}(3 b+a-2)$ \\
2 & $\frac{1}{2}(b+a-1)$ & -2 \\
1 & -1 & 1 \\
0 & 1 & 0 \\
-1 & 1 & 1 \\
-2 & $\frac{1}{2}(b+a-1)$ & 2 \\
-3 & $\frac{1}{2}(3 a+b-2)$ & $\frac{1}{2}(3 b+a-2)$ \\
-4 & $\frac{1}{2}(b+a+1)(b+a-3)-\frac{1}{4}(b-a+3)(b-a-3)$ & $2(b+a-1)$ \\
-5 & $(b+a-4)^{2}-\frac{1}{4}(b-a-4)^{2}-\frac{1}{2}(b+a-4)(b-a-4)+$ & $(b+a-4)^{2}-\frac{1}{4}(b-a-4)^{2}+\frac{1}{2}(b+a-4)(b-a-4)+$ \\
& $4(b+a-4)-\frac{7}{2}(b-a-4)$ & $8(b+a-4)-\frac{1}{2}(b-a-4)+12$ \\
\hline
\end{tabular}

$$
\begin{aligned}
= & \frac{\Gamma\left(\frac{1}{2}\right) \Gamma\left(\frac{1}{2}\left(a+\mu_{1}+\cdots+\mu_{r}+\ell+1\right)\right) \Gamma\left(\frac{1}{2}\left(\mu_{1}+\cdots+\mu_{r}\right)-\frac{a}{2}-\frac{\ell}{2}+\frac{1}{2}\right)}{\Gamma\left(\frac{1}{2}\left(\mu_{1}+\cdots+\mu_{r}\right)-\frac{a}{2}+\frac{|\ell|}{2}+\frac{1}{2}\right)} \\
& \cdot \sum_{n=0}^{\infty} \frac{\left(\frac{a}{2}\right)_{n}\left(\frac{a}{2}+\frac{1}{2}\right)_{n}\left(\mu_{1}\right)_{n}\left(\mu_{2}\right)_{n}}{(\alpha)_{n}\left(\frac{1}{2}\left(a+\mu_{1}+\cdots+\mu_{r}+\ell+1\right)\right)_{2 n} n !} \\
& \cdot\left[\frac{A_{\ell}}{\Gamma\left(\frac{1}{2}\left(\mu_{1}+\cdots+\mu_{r}+1\right)\right) \Gamma\left(\frac{a}{2}+\frac{\ell}{2}+\frac{1}{2}-\left[\frac{\ell+1}{2}\right]\right)\left(\frac{1}{2}\left(\mu_{1}+\cdots+\mu_{r}+1\right)\right)_{n}}\right. \\
& \cdot \frac{1}{\left(\frac{a}{2}+\frac{\ell}{2}+\frac{1}{2}-\left[\frac{\ell+1}{2}\right]\right)_{n}}+\frac{B_{\ell}}{\Gamma\left(\frac{1}{2}\left(\mu_{1}+\cdots+\mu_{r}\right)\right) \Gamma\left(\frac{a}{2}+\frac{\ell}{2}-\left[\frac{\ell}{2}\right]\right)} \\
& \left.\cdot \frac{1}{\left(\frac{1}{2}\left(\mu_{1}+\cdots+\mu_{r}\right)\right)_{n}\left(\frac{a}{2}+\frac{\ell}{2}-\left[\frac{\ell}{2}\right]\right)_{n}}\right]
\end{aligned}
$$

where $\ell=0, \pm 1, \pm 2, \pm 3, \pm 4, \pm 5$ and, here, the coefficients $A_{\ell}$ and $B_{\ell}$ can be obtained in replacing $a$ and $b$ in Table 1 by $\mu_{1}+\cdots+\mu_{r}+2 n$ and $a+2 n$, respectively.

Proof The proof is quite straightforward. In fact, if we set $x=\frac{1}{2}$ and $b=\frac{1}{2}\left(a+\mu_{1}+\cdots+\right.$ $\left.\mu_{r}+\ell+1\right)$ in Equation (1.8), we have the following form:

$$
\begin{aligned}
& F_{1: 1 ; 1 ; 0 ; \ldots ; 0 ; 0}^{2: 1: 1 ; 1 ; \ldots ;}\left(\begin{array}{r}
(a: 1, \ldots, 1),(\alpha: 1,1,0, \ldots, 0): \\
\left(\frac{1}{2}\left(a+\mu_{1}+\cdots+\mu_{r}+\ell+1\right): 1, \ldots, 1\right):
\end{array}\right.
\end{aligned}
$$

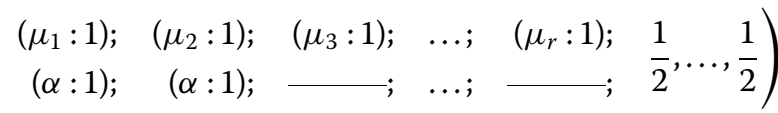

$$
\begin{aligned}
& =\sum_{n=0}^{\infty} \frac{(a)_{2 n}\left(\mu_{1}\right)_{n}\left(\mu_{2}\right)_{n}}{(\alpha)_{n}\left(\frac{1}{2}\left(a+\mu_{1}+\cdots+\mu_{r}+\ell+1\right)\right)_{2 n} 2^{2 n} n !} \\
& \cdot{ }_{2} F_{1}\left[\begin{array}{rr}
\mu_{1}+\cdots+\mu_{r}+2 n, a+2 n ; & 1 \\
\frac{1}{2}\left(a+\mu_{1}+\cdots+\mu_{r}+\ell+1+4 n\right) ; & 2
\end{array}\right] .
\end{aligned}
$$

Now, we observe that the ${ }_{2} F_{1}$ appearing on the right-hand side of (2.2) can be evaluated with the help of generalized Kummer's second summation theorem (1.11) in replacing $a$ and $b$ by $\mu_{1}+\cdots+\mu_{r}+2 n$ and $a+2 n$, respectively. And, after a little simplification, we 
easily arrive at the right-hand side of our main formula (2.1). The completes the proof of Theorem 2.

\section{Special cases}

It is easy to see that the special case of (2.1) when $\ell=0$ leads to Equation (1.10) due to Jaimini et al. [11]. Here we consider two interesting special cases of our main formula (2.1). Setting $\ell=-1$ and $\ell=1$ in (2.1), we find Equations (3.1) and (3.2), respectively:

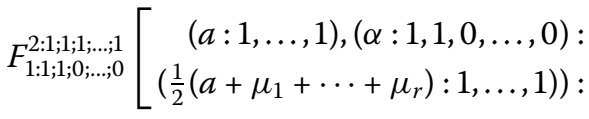

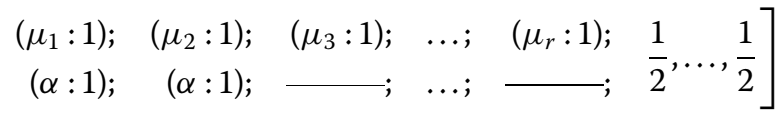

$$
\begin{aligned}
& =\Gamma\left(\frac{1}{2}\right) \Gamma\left(\frac{1}{2}\left(a+\mu_{1}+\cdots+\mu_{r}\right)\right) \\
& \cdot\left(\frac{1}{\Gamma\left(\frac{a}{2}\right) \Gamma\left(\frac{1}{2}\left(\mu_{1}+\cdots+\mu_{r}+1\right)\right)}{ }_{3} F_{2}\left[\begin{array}{rr}
\frac{a}{2}+\frac{1}{2}, \mu_{1}, \mu_{2} ; & 1 \\
\alpha, \frac{1}{2}\left(\mu_{1}+\cdots+\mu_{r}+1\right) ; & 1
\end{array}\right]\right. \\
& \left.+\frac{1}{\Gamma\left(\frac{a}{2}+\frac{1}{2}\right) \Gamma\left(\frac{1}{2}\left(\mu_{1}+\cdots+\mu_{r}\right)\right)}{ }_{3} F_{2}\left[\begin{array}{rr}
\frac{a}{2}, \mu_{1}, \mu_{2} ; & 1]) \\
\alpha, \frac{1}{2}\left(\mu_{1}+\cdots+\mu_{r}\right) ; &
\end{array}\right]\right)
\end{aligned}
$$

and

$$
\begin{aligned}
& F_{1: 1 ; 1 ; 0 ;, \ldots, 0}^{2: 1: 1 ; 1 ; \ldots ; 1}\left[\begin{array}{r}
(a: 1, \ldots, 1),(\alpha: 1,1,0, \ldots, 0): \\
\left.\left(\frac{1}{2}\left(a+\mu_{1}+\cdots+\mu_{r}+2\right): 1, \ldots, 1\right)\right):
\end{array}\right. \\
& \begin{array}{rrrrr}
\left(\mu_{1}: 1\right) ; & \left(\mu_{2}: 1\right) ; \quad\left(\mu_{3}: 1\right) ; & \ldots ; & \left(\mu_{r}: 1\right) ; & 1 \\
(\alpha: 1) ; & (\alpha: 1) ; \quad \longrightarrow, \frac{1}{2}
\end{array} \\
& =\frac{2 \Gamma\left(\frac{1}{2}\right) \Gamma\left(\frac{1}{2}\left(a+\mu_{1}+\cdots+\mu_{r}+2\right)\right)}{\mu_{1}+\cdots+\mu_{r}-a} \\
& \cdot\left(\frac{1}{\Gamma\left(\frac{a}{2}+\frac{1}{2}\right) \Gamma\left(\frac{1}{2}\left(\mu_{1}+\cdots+\mu_{r}\right)\right)}{ }_{3} F_{2}\left[\begin{array}{rr}
\frac{a}{2}+\frac{1}{2}, \mu_{1}, \mu_{2} ; & 1 \\
\alpha, \frac{1}{2}\left(\mu_{1}+\cdots+\mu_{r}\right) ; &
\end{array}\right]\right. \\
& \left.-\frac{1}{\Gamma\left(\frac{a}{2}\right) \Gamma\left(\frac{1}{2}\left(\mu_{1}+\cdots+\mu_{r}\right)+1\right)}{ }_{3} F_{2}\left[\begin{array}{rr}
\frac{a}{2}, \mu_{1}, \mu_{2} ; & 1 \\
\alpha, \frac{1}{2}\left(\mu_{1}+\cdots+\mu_{r}\right)+1 ; &
\end{array}\right]\right) \text {. }
\end{aligned}
$$

Clearly Equations (3.1) and (3.2) are closely related to Equation (1.10). The other special cases of (2.1) can also be obtained.

\section{Competing interests}

The authors declare that they have no competing interests.

\section{Authors' contributions}

The authors have equal contributions to each part of this paper. All authors have read and approved the final manuscript.

\section{Author details}

'Department of Mathematics, Dongguk University, Gyeongju, Korea. ${ }^{2}$ Department of Mathematics, School of Mathematical \& Physical Sciences, Central University of Kerala, Riverside Transit Campus, Padennakad P.O. Nileshwar, Kasaragod, 671 328, India. 


\section{Acknowledgements}

This paper was supported by the Basic Science Research Program through the National Research Foundation of Korea funded by the Ministry of Education, Science and Technology (2010-0011005)

Received: 12 March 2013 Accepted: 14 June 2013 Published: 3 July 2013

\section{References}

1. Rainville, ED: Special Functions. Macmillan Company, New York (1960) Reprinted by Chelsea Publishing Company, Bronx, New York (1971)

2. Srivastava, HM, Choi, J: Zeta and q-Zeta Functions and Associated Series and Integrals. Elsevier, Amsterdam (2012)

3. Srivastava, HM, Karlsson, PW: Multiple Gaussian Hypergeometric Series. Wiley, New York (1985)

4. Choi, J, Rathie, AK: Relations between Lauricella's triple hypergeometric function $F_{A}^{(3)}(x, y, z)$ and Exton's function $X_{8}$. Adv. Differ. Equ. 2013, 34 (2013). doi:10.1186/1687-1847-2013-34

5. Srivastava, HM: On the reducibility of Appell's function $F_{4}$. Can. Math. Bull. 16, 295-298 (1973)

6. Srivastava, HM: Some generalizations of Carlson's identity. Boll. Unione Mat. Ital., A 18(5), 138-143 (1981)

7. Buschman, RG, Srivastava, HM: Series identities and reducibility of Kampé de Fériet functions. Math. Proc. Camb. Philos. Soc. 91, 435-440 (1982)

8. Grosjean, CC, Sharma, RK: Transformation formulae for hypergeometric series in two variables (II). Simon Stevin 62 , 97-125 (1988)

9. Grosjean, CC, Srivastava, HM: Some transformation and reduction formulas for hypergeometric series in several variables. J. Comput. Appl. Math. 37, 287-299 (1991)

10. Srivastava, HM, Raina, RK: Some combinatorial series identities. Math. Proc. Camb. Philos. Soc. 96, 9-13 (1984)

11. Jaimini, BB, Koul, CL, Srivastava, HM: Some multiple series identities. Comput. Math. Appl. 28(4), 19-24 (1994)

12. Bailey, WN: Generalized Hypergeometric Series. Cambridge Tracts in Mathematics and Mathematical Physics, vol. 32. Cambridge University Press, Cambridge (1935). Reprinted by Stechert-Hafner Service Agency, New York and London (1964)

13. Choi, J, Rathie, AK, Srivastava, HM: A generalization of a formula due to Kummer. Integral Transforms Spec. Funct. 22 851-859 (2011)

14. Lavoie, JL, Grondin, F, Rathie, AK: Generalizations of Whipple's theorem on the sum of a ${ }_{3} F_{2}$. J. Comput. Appl. Math. 72, 293-300 (1996)

doi:10.1186/1029-242X-2013-312

Cite this article as: Choi and Rathie: Reduction formulae for the Lauricella functions in several variables. Journal of Inequalities and Applications 2013 2013:312.

\section{Submit your manuscript to a SpringerOpen ${ }^{\ominus}$ journal and benefit from:}

- Convenient online submission

- Rigorous peer review

- Immediate publication on acceptance

- Open access: articles freely available online

- High visibility within the field

- Retaining the copyright to your article 